\title{
Is Any Correlation between Platelet Indices with Extent of Coronary Artery Involvement in Ischemic Heart Diseases?
}

\section{Mohammad Hassan Adel ${ }^{*}$, Masood Seyedian'1, Marjan Jafarsalehi' ${ }^{1}$, Mohammad Hossein Najafi ${ }^{1}$, Mohammad Nourizadeh', Mehdi Mohebi², Sara Nourizadeh ${ }^{3}$}

\author{
${ }^{1}$ Atherosclerosis Research Center, Jundishapur University of Medical Sciences, Ahvaz, Iran \\ ${ }^{2}$ Department of Medical Genetics, Faculty of Medicine, Tehran University of Medical Sciences, Tehran, Iran \\ ${ }^{3}$ Department of Biochemistry, Kharazmi University, Tehran, Iran \\ Email: ‘jafasalehi_marjan@yahoo.com, dr.hassan.adel@gmail.com
}

How to cite this paper: Adel, M.H., Seyedian, M., Jafarsalehi, M., Najafi, M.H., Nourizadeh, M., Mohebi, M. and Nourizadeh, S. (2016) Is Any Correlation between Platelet Indices with Extent of Coronary Artery Involvement in Ischemic Heart Diseases? Health, 8, 1266-1274.

http://dx.doi.org/10.4236/health.2016.812129

Received: June 21, 2016

Accepted: September 24, 2016

Published: September 27, 2016

Copyright $\odot 2016$ by authors and Scientific Research Publishing Inc. This work is licensed under the Creative Commons Attribution International License (CC BY 4.0).

http://creativecommons.org/licenses/by/4.0/ (c) (i) Open Access

\begin{abstract}
Ischemic heart disease (IHD) is the most common cause of death around the world. Nowadays Platelet counts (PC) and volumetric platelet indices are available routinely in most laboratories and reflect the level of mobility and production of platelets. It seems that the excessive flexibility and size of the platelets and their local activation have correlation with extent of ischemic heart disease. So our objective is the study of platelet indices in ischemic heart disease. This non-randomized prospective study was performed on 245 patients with ischemic heart disease, who underwent the coronary angiography. The patients were divided into four groups: stable angina, unstable angina, acute myocardial infarction and control group; and then platelet indices, including the platelet counts (PC), the average platelet volume (MPV), the Platelet Distribution Width (PDW) and plateletcrit (PCT) in each group with the extent of coronary disease were compared based on an Syntax Score system and observational methods. The average ages of the patients were 57 years and $65 \%$ of them were male and the rest were female. A significant difference is exists between indices in all three groups compared to the control that this difference was related to gender and the type of the coronary artery involvement. However, only in infarction group, PDW in different disease intensities was significantly different. In this study, unlike many of the previous studies no relationship was found between the MPV with the extent of coronary disease.
\end{abstract}

\section{Keywords}

Ischemic Heart Disease, Platelet Indices, Average Platelet Size, Platelet Distribution 


\section{Introduction}

Ischemic heart disease is one of the common causes of mortality in the entire world; also, according to the Framingham's studies, the risk of coronary artery disease after age of 40 in men and women is respectively $45 \%$ and $32 \%$ [1].

Increased activation of the platelets happens before coronary events. Increased consumption of platelets at the site of the coronary atherosclerotic plaque causes larger platelets to be free of the marrow. It is familiar fact that an increase in the size of the platelets after discharge also remains stable supports an increase in the risk of reinfarction. MPV is an index of mobility and production of platelets. MPV may be a marker for restenosis after angioplasty. MPV had a correlation with type II diabetes and microvascular involvement.

According to the findings of the AHA, among the platelet parameters the PDW (and no platelet counts and MPV) is an independent predictor of mortality (heart and any cause) and routine measurement of these in all patients is recommended [2].

Automatic measurement of platelet count has been replaced for manual count. This led to getting simple and to improve the accuracy of the measurement and the considerable time-saving. Due to the greater number of cells with this method is checked, a statistical error has considerably been reduced.

In more hematology analyzers, a platelet count can be done by measuring the impedance. With the addition of the supplementary methods, role of confounding factors has been also very light [3]. It seems that abnormal platelets indices may be as risk factors for coronary artery disease.

Due to the ease of doing, to be available and affordable costs, proof of the relationship between the platelet indices with the extent of coronary artery disease can be an important step in improving risk assessment and screening for high risk patients, in order to perform more aggressive investigations.

In this regard, the study has been designed in order to investigate the relationship between the platelet indices with the extent of coronary disease among different groups through a simultaneous review of angiography findings with Syntax Score and observational systems.

\section{Materials and Methods}

This study was prospective and planned based on hospital data. Patients were selected non-randomly and the subjects were divided into four groups; the first group: 76 patients with stable angina who have been referred for angiography. The second group: 63 patients who have been hospitalized in an emergency with a diagnosis of unstable angina.The third group: 62 patients hospitalized due to acute myocardial infarction. The fourth group (the control group): healthy subjects with normal ECG and echocardiography. In this study; 201 patients with 44 healthy were studied as a control group; over the course of the research. 8 patients were omitted from study because insufficient and unreliable data.

Platelet indices, including the PDW, MPV, PCT and the number of platelets in the 
patients' records based on the CBC (based on sample arrival) were collected and after performing angiography, their relationships were investigated. Age, sex, changes in ECG and taking anti-platelet drug were recorded in order to control platelet antibodies as well as underlying and confounding factors. According to the contradictory findings of previous studies about the impact of risk factors in the indices, the risk factors were investigated in all patients. As well as preventing the bias in the study groups, this will be done in terms of matching.

\subsection{Inclusion Criteria}

1) All patients admitted with a diagnosis of acute coronary syndrome (ACS) and according to the existing indications in the guideline, will be undergoing angiography.

2) All patients who are hospitalized for elective angiography.

\subsection{Exclusion Criteria}

1) Patients with a history of the previous revascularization, coronary arteritis bypass graft (CABG) and percutaneous coronary intervention (PCI)

2) A history of blood and platelet products received during the past three months

3) A history of cancer, and chemotherapy

4) Consumption of drugs with hematological complication.

5) History of platelet diseases, including idiopathic thrombocytopenia (ITP), thrombotic thrombocytopenic purpura (TTP)

6) Severe and an acute hemorrhage.

The data were collected using the patients' records and on the basis of the information contained in it. Sysmex, the model KX21N for platelet indices and SYNTAX software score system for angiography to check the extent of CAD. Selective angiography was performed by Seimens model Zee Floor 2012 by expert cardiologist. Coronary angiography results were interpreted by two cardiologists according to observational methods and with SYNTAX software separately.

\subsection{Statistical Analysis}

Data collection and analysis was performed by self-administer questionaries' and SPSS software 16. Baseline data are reported as means \pm standard deviation (SD) and categorical data are analyzed by Chi-square, independent sample test or non-parametric analysis, univariate ANOVA and welch analysis. Cox proportional-hazards modeling was used for efficacy and safety analyses. A P value of 0.05 or less was considered to indicate statistical significance.

\section{Results}

The study compared 201 patients in three groups: stable angina, unstable angina (UA) and myocardial infarction (MI) with a control group of 44 patients with platelet indices. The average age of the study patients in the unstable angina, angina-stable, and myocardial infarction groups was 57.2, 57.7 and 56 years, respectively. About $35 \%$ of patients 
were female. $21.3 \%$ patients showed a brief involvement of coronary; $24.2 \%$ showed involvement of a vessel; $20.3 \%$, the involvement of two vessels; and the rest, all three coronary vessels. Average number of platelets, PCT, MPV, PDW, was respectively 239 $\times 103 \pm 57,15.7 \pm 0.55,10.1 \pm 1.81$ and, $249 \pm 0.07$ in patients with stable angina. Average number of platelets in patients with UA and MI was reported respectively $249 \times$ $103 \pm 61.7$ and, $261 \times 103 \pm 67$; PDW $15.1 \pm 1.1$ and $15.3 \pm 1.2$; MPV $11 \pm 1.1$ and 10.4 \pm 1.4 and PCT $0.275 \pm 0.07$ and $0.272 \pm 0.07$ (Table 1 ).

About $30.3 \%, 12.7 \%$ and $3.2 \%$ of patients with respectively stable angina, unstable angina, and myocardial infarction had the consumption history of antiplatelet drugs. The average hemoglobin in the three groups above was respectively $12.5 \pm 1.6,12.3 \pm$ 1.6 , and $13.4 \pm 1.8$ and the average left ventricular ejection fraction was $46.8,43.6$, and $39.1 \%$ respectively.

Platelet indices were analyzed based on extent of coronary artery disease (with an observational method and syntax score system) in the studied groups. Total data obtained were analyzed again using univariate ANOVA to investigate the correlation between indices with the extent of coronary artery disease. Analysis of variance was

Table 1. Baseline demographic and laboratory characteristics of patients.

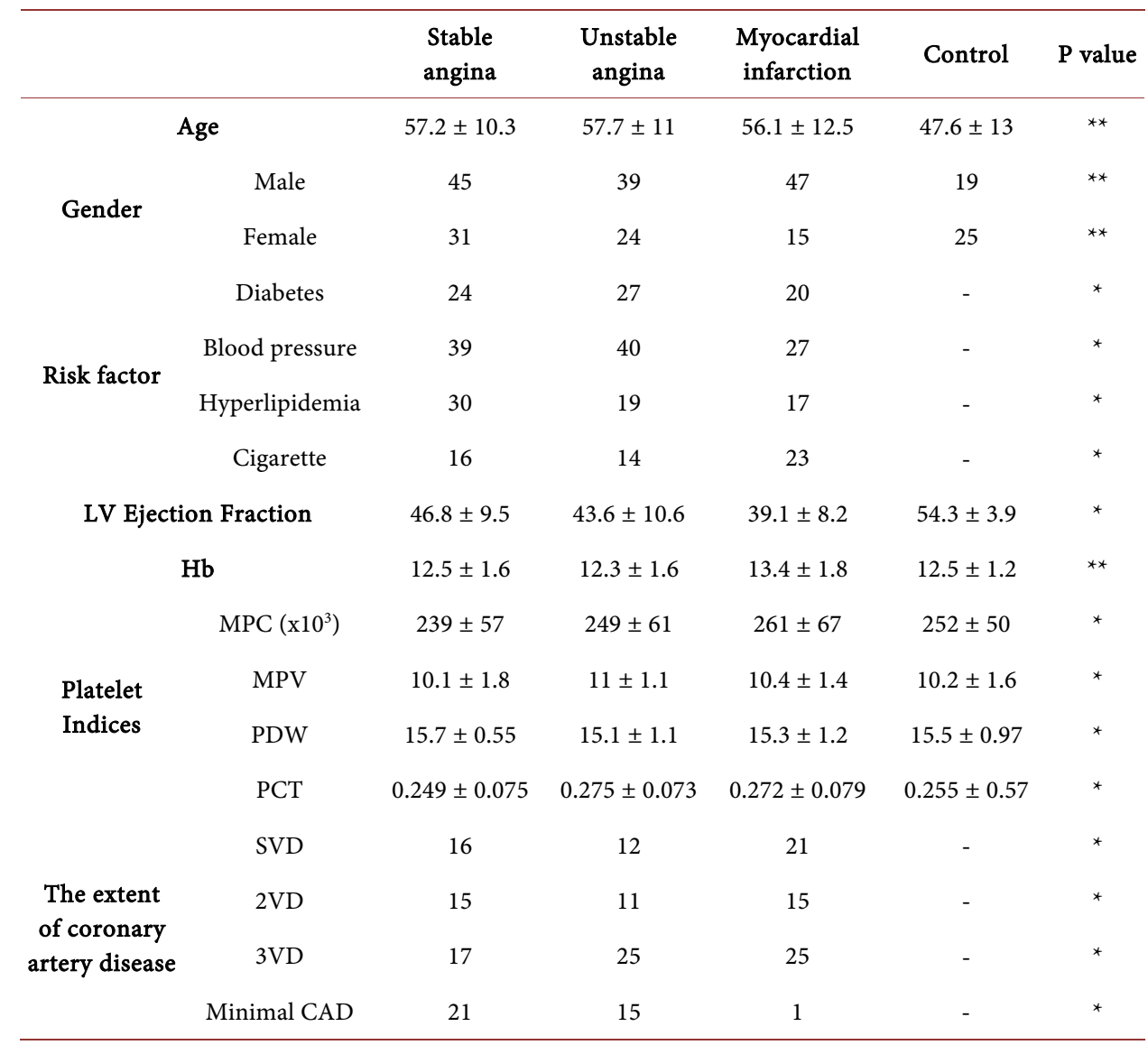

Hemoglobin ( $\mathrm{Hb}$ ); Mean Platelet Counts (MPC), Average Platelet Volume (MPV), Platelet Distribution Width (PDW) and Plateletcrit (PCT), Single Vessel Diseases (SVD); Two Vessel Diseases (2VD); Three Vessel Diseases (3VD); Minimal Coronary Artery Diseases (Minimal CAD), ${ }^{*} \mathrm{p}$ value $<0.05,{ }^{* *} \mathrm{p}$ value $>0.05$. 
performed to evaluate the average age in four groups. Welch analysis used for lack of homogeneity of variance in the four groups. The chi square test determined the sex of the patients is different in the four groups; therefore, we entered the age and sex as confounding variables into the model.

The results showed although gender has the confounding effects in the model ( $\mathrm{p}=$ 0.001 ), the average platelet in patients with stable angina and unstable angina is statistically lower than control group and higher than myocardial infarction $(\mathrm{p}=0.002)$. In different groups average of MPV have significant statistical difference with control group $(p=0.041)$ that is due to type of diseases $(p=0.012)$ and the age and sex had not confounding effect on this variable.

The average PDW in different disease severities in patients with stable angina and unstable angina and myocardial infarction were compared with control group ( $\mathrm{p}=$ 0.030) which this effect is related to the severity of the disease. The results of analysis of variance showed that the average PDW is the same in different disease intensities ( $\mathrm{p}=$ 0.016).

With regard to the possibility of the confounding effect of risk factors in getting significant this index, analysis was conducted to examine the effect of them again, that only diabetes had a significant impact and this difference was not significant in other risk factors $(\mathrm{p}=0.019)$.

Platelets indices were analyzed with extent of coronary involvement by observational methods (Table 2). It showed mean platelets count in stable angina groups with three vessel diseases was higher than mono vessel diseases $(p=0.037)$ and sex had confounding effect of this finding $(\mathrm{p}=0.022)$.

Table 2. Mean of platelet indices in different extent of coronary involvement by observational methods among patients groups.

\begin{tabular}{ccccc}
\hline & MPC & PCT & MPV & PDW \\
\hline Mono vessel involvement & & & & \\
Stable angina & $70^{* 1} \pm 248$ & $0.07^{* 3} \pm 0.244$ & $1.5 \pm 10$ & $0.24 \pm 15.7$ \\
Unstable angina & $72 \pm 239$ & $0.07 \pm 0.260$ & $1.1 \pm 10.8$ & $0.9 \pm 15.4$ \\
Myocardial infarction & $84 \pm 279$ & $0.10 \pm 0.282$ & $1.6 \pm 9.9$ & $1.6^{* 2} \pm 14.9$ \\
Two vessel involvement & & & & \\
Stable angina & $48 \pm 242$ & $0.05^{* 3} \pm 0.252$ & $1.6 \pm 10.5$ & $0.3 \pm 15.7$ \\
Unstable angina & $55 \pm 250$ & $0.06 \pm 0.276$ & $1.5 \pm 11.2$ & $0.9 \pm 15.2$ \\
Myocardial infarction & $55 \pm 258$ & $0.06 \pm 0.281$ & $0.9 \pm 10.9$ & $1.1 \pm 15.1$ \\
Three vessel involvement & & & & \\
Stable angina & $37^{* 1} \pm 201$ & $0.08^{* 3} \pm 0.216$ & $1.3 \pm 9.9$ & $0.3 \pm 15.8$ \\
Unstable angina & $72 \pm 262$ & $0.08 \pm 0.293$ & $1.2 \pm 11$ & $1.5 \pm 14.9$ \\
Myocardial infarction & $58 \pm 249$ & $0.05 \pm 0.259$ & $1.5 \pm 10.5$ & $0.4^{* 2} \pm 15.8$ \\
\hline
\end{tabular}

Mean Platelet Counts (MPC), Average Platelet Volume (MPV), Platelet Distribution Width (PDW) and Plateletcrit $(\mathrm{PCT}),{ }^{* 1} \mathrm{p}$ value $=0.037 ;{ }^{* 2} \mathrm{p}$ value $=0.041 ;{ }^{* 3} \mathrm{p}$ value $=0.004$. 
Mean platelet scout was not different in unstable angina and myocardial infarction with different intensity of vessel involvement. Mean MPV in three groups with different intensity were not statistically different. Mean PDW was not statistically different in different intensity among unstable and stable angina but was significantly different in three vessel diseases with myocardial infarction $(\mathrm{p}=0.041)$. Analysis by post HOC (Tukey HSD) was showed that age and sex was not confounding effect. Due to confounding effect of sex $(p=0.001)$, mean PCT was statically different in stable angina with two vessel than single and three vessels $(\mathrm{p}=0.004)$. Mean PCT among unstable angina and myocardial infarction was not different. Results of indices analysis based on SYNTAX score showed that mean platelets number in stable angina with SYNTAX score $=10-20$ and SYNTAX score $>20$ was lower in males than patients with SYNTAX score $<10(\mathrm{p}=0.02)$ (Table 3$)$. The PDW in patients with unstable angina in SYNTAX score $=10-20$ and SYNTAX score $>20$ were significantly different than SYNTAX score $<10$ among females $(\mathrm{p}=0.02)$. The PDW in patients with Myocardial infarction with SYNTAX score $=10-20$ and SYNTAX score $>20$ were significantly different than SYNTAX score $<10$ among males $(\mathrm{p}=0.04)$.

\section{Discussion}

In the developed countries most deaths are associated with CAD and platelets play a fundamental role in atherosclerosis and thrombosis [2]. Great variability exists in initial reactivity of platelets that potentially may be resulted from differences in their sizes. In fact, the larger platelets have more mass and metabolically and enzymatically are more

Table 3. Mean of platelet indices in different extent of coronary involvement by Syntax Score System among patients groups.

\begin{tabular}{ccccc}
\hline & MPC & PCT & MPV & PDW \\
\hline Syntax $<10$ & & & & \\
Stable angina & $59^{* 1} \pm 257$ & $0.08 \pm 0.268$ & $1.7 \pm 10.2$ & $0.6 \pm 15.6$ \\
Unstable angina & $51 \pm 238$ & $0.05 \pm 0.259$ & $1.2 \pm 10.8$ & $0.7^{\star 2} \pm 15.4$ \\
Myocardial infarction & $83 \pm 271$ & $0.10 \pm 0.282$ & $1.5 \pm 10.4$ & $1.5^{\star 3} \pm 15$ \\
Syntax: 10 - 20 & $41^{* 1} \pm 209$ & $0.05 \pm 0.222$ & $2.3 \pm 10$ & $0.3 \pm 15.7$ \\
Stable angina & $49 \pm 253$ & $0.06 \pm 0.280$ & $1.1 \pm 10.8$ & $1.6^{\star 2} \pm 14.6$ \\
Unstable angina & $57 \pm 264$ & $0.06 \pm 0.274$ & $1.6 \pm 10.5$ & $1^{* 3} \pm 15.4$ \\
Myocardial infarction & & & & \\
Syntax $>$ 20 & $58 \pm 267$ & $0.10 \pm 0.302$ & $1.1 \pm 11.3$ & $1^{\star 2} \pm 15.4$ \\
Stable angina & $43 \pm 237$ & $0.04 \pm 0.248$ & $1 \pm 10.5$ & $0.5^{* 3} \pm 15.8$ \\
\hline Unstable angina & & & & \\
Myocardial infarction & & & & \\
\hline
\end{tabular}

Mean Platelet Counts (MPC), Average Platelet Volume (MPV), Platelet Distribution Width (PDW) and Plateletcrit $(\mathrm{PCT}),{ }^{* 1} \mathrm{p}$ value $=0.02 ;{ }^{* 2} \mathrm{p}$ value $=0.02:{ }^{* 3} \mathrm{p}$ value $=0.02$. 
active than small platelets. These platelets due to higher levels of thromboxane A2 and BTG as well as increased intracellular and surface procoagulant protein have more prothrombotic properties. Homeostatically, reactive platelets were larger, and the granules and receptors have more adhesion. This makes bleeding time (BT) longer in tests that shows increased platelet activation. Analysis between the groups showed that an average of every four Platelet indices in three study groups was significantly different compared to the control group.

Average MPV in patients with severe coronary artery $10.4 \pm 1.37$ was higher than a study conducted in Turkey [4] and America [3]. In this study the relationship between an MPV and extent of CAD based on SYNTAX Score was not observed, but a similar study in Turkey [4] reported a positive relationship between them. In this study, a positive relationship between the amount of PDW and extent CAD based on both methods was examined and the extent of CAD was obtained, but in a study in Italy [5], no relationship found between them. Platelets average a difference among the groups was due to the effect of gender and it was not associated with disease. This finding is consistent with the results of a study conducted by Khanderkar et al. [6] in India.The effect of the disease type on the MPV and PDW was similar to the results of a study conducted by Ihara et al. [7] and Tavil et al. [8]. In a cohort study on 1401 patients, a relationship was observed between MPV, platelet aggregation, carotid intima thickness and extent of CAD [9]. Similar results were obtained in studies conducted by Tavil et al. [10] and Halbmayer et al. [11]. PDW indicates changes in platelet size and will provide us with useful information compared with the MPV that may indicate the number of reactive platelets. Increased MPV may be due to increased production of platelets in the marrow that causes larger reticulated platelets to release into the blood stream. In a study, PDW was inversely associated with the number of platelets which can be suggestive use of smaller platelets and a compensatory increase in large reticulated platelets. Indeed MPV is associated with megakaryocyte plyoid and the percentage of circulating reticulated platelets. Thus, higher MPV may not suggest greater platelet reactivity and its relationship to the extent and complexity of CAD. In this study no relationship between MPV and extent of CAD was observed in any of the groups studied [5]. MPV is associated with other prognostic factors such as smoking [12], diabetes [13], obesity [14], which may affect initially clinical extent and outcome of CAD. In a study in India [6], PVI (Platelet volume indices) in patients with ACS was increased compared to controls and patients with stable angina. This result is in agreement with the results of other studies and this study. Generalized platelet activation occurs before an acute coronary event. Consumption of platelets at the site of atherosclerotic plaque leads to the release of larger platelets from the marrow. The fact that this increase even after patient discharge time goes on, is indicating a larger volume of chronic platelets in patient with infarction [15] [16]. This suggests that PVI and especially MPV is an indicator of the severity of injury created and these markers maintain their prospective power and value for a long time. But no relationship between elapsed time from MI and MPV has been found in some studies. This group also believes that the MPV does not change during the acute 
phase and is determined by other factors [17]. In a study in India using analysis of logistic regression, values of MPV $>9.6 \mathrm{fl}$ were associated with the notable risk of the incidence of MI in patients with CAD while Endler et al. reported values of MPV > 6/11fl. In this study no relationship was observed between PVI with morbidity and mortality, whereas in the study by Martin et al., the MPV was significantly higher in patients who had died [6].

\section{Conclusion}

Our findings suggest that the MPV is associated with a pre-thrombotic state in acute ischemic syndromes and larger platelets may have a special role in the infarct. Patients with larger platelets are easily identifiable during hematologic evaluations. PVI is a major, simple and affordable tool that can be used to predict impending MI [6]. Results show the measure of platelet indices may reflect the underlying pathophysiological conditions and subsequent clinical events in patients with IHD. The low P-LCR seems particularly effective in identifying patients with positive angiographies [7]. Lippi et al. showed that MPV in patients with ACS in hospitalization compared with patients with non-cardiac angina is higher [18]. At all this study showed that the relationship between platelet indices with extent of the $\mathrm{CAD}$, and thus we did not find clear relationship between MPV with extent of coronary involvement and many relation may be due to effect of sex. Therefore, taking into account other risk factors as the confounding variables, comment in this regards is better to postpone to another study.

\section{Recommendations}

In the study the information contained in the files and based on the first sample of the $\mathrm{CBC}$ taken from a patient on admission to the emergency or ward was used; as a result the samples have been taken by different people and at different time interval of occurrence of an accident (especially in acute coronary syndromes) and the technical differences and the effect of time has not been removed. On the other hand, analysis was done with consideration of the confounding effect of the age and gender variables. It seems that the design of a study is reasonable by taking all the underlying conditions for more accurate conclusion. In total the results of this study consistent with often earlier surveys confirmed the relationship between coronary artery disease and platelet indices, and demonstrated that a marker from the more activation accordingly a wider disease can be in patients with myocardial infarction. According to be high these indices after revascularization, it may be used to assess risk after partial revascularization. By controlling all of the underlying factors in more reviews and studies and possibly in the future these indices will be considered as coronary risk factors.

\section{References}

[1] Katdare, A. and Kakrani, A. (2009) Prevalence of IHD among Urban Population of Siliguri, West Bengal. Indian Journal of Community Medicine, 34, 19-23.

http://dx.doi.org/10.4103/0970-0218.44518 
[2] Assiri, A., Jamil, A., Ghallab, M., et al. (2012) Diagnostic Importance of Platelet Parameters in Patients with Acute Coronary Syndrome Admitted to a Tertiary Care Hospital in Southwest Region, Saudi Arabia. Journal of The Saudi Heart Association, 24, 17-21.

[3] Qayyam, R. and Vaidya, D. (2011) Platelet Distribution Width Is an Independent Predictor of All-Cause and Cardiovascular Mortality among Healthy U.S Adults. Circulation, 124, 16788.

[4] Berkay, E., Aycan, F., Aslihan, A., et al. (2013) Is Average Platlet Volume Associated with the Angiographic Severity of Coronary Artery Disease? Kardiologia Polska, 8, 832-838.

[5] De Luca, G., Venegoni, L., Iorio, S., et al. (2010) Platelet Distribution Width and the Extent of Coronary Artery Disease: Results from a Large Prospective Study. Platelets, 21, 508-514. http://dx.doi.org/10.3109/09537104.2010.494743

[6] Khandekar, M., Khurana, A., Deshmukh, S., et al. (2006) Platelet Volume Indices in Patients with Coronary Artery Disease and Acute Myocardial Infarction: On Indian Scenario. Journal of Clinical Pathology, 59, 146-149. http://dx.doi.org/10.1136/jcp.2004.025387

[7] Ihara, A., Kawamoto, T., Matsumoto, K., et al. (2006) Relationship between Platelet Indexes Coronary Angiographic Findings in Patients with Ischemic Heart Disease. Pathophysiology of Haemostasis and Thrombosis, 35, 376-379. http://dx.doi.org/10.1159/000097692

[8] Tavil, Y., Sen, N., Yazici, H., et al. (2010) Coronary Heart Disease Is Associated with Average Platelet Volume in Type 2 Diabetic Patients. Platelets, 21, 368-372.

http://dx.doi.org/10.3109/09537101003628421

[9] Jenning, L.K. (2009) Role of Platelets in Atherothrombosis. American Journal of Cardiolo$g y$, 103, 4A-10A. http://dx.doi.org/10.1016/j.amjcard.2008.11.017

[10] Sen, N., Tavil, Y., Yazici, H.U., et al. (2007) Average Platelet Volume in Patients with Coronary Artery Ectasia. Medical Science Monitor, 13, 356-359.

[11] Coban, E., Bostan, E. and Ozdogan, M. (2006) The Average Platelet Volume in Subjects with Impaired Fasting Glucose. Platelets, 17, 67-69. http://dx.doi.org/10.1080/09537100500220729

[12] Hekimsoy, Z., Payzin, B., Ornek, T., et al. (2004) Average Platelet Volume in Type 2 Diabetic Patients. Journal of Diabetes and its Complications, 18, 173-176.

http://dx.doi.org/10.1016/S1056-8727(02)00282-9

[13] Brown, A.S., Hong, Y., Beacon, H., et al. (1997) Mega Kariocyte Ploidy and Platelet Changes in Human Diabetes and Atherosclerosis. Arteriosclerosis, Thrombosis, and Vascular Biology, 17, 802-807. http://dx.doi.org/10.1161/01.ATV.17.4.802

[14] Sharpe, P.C. and Trinick, T.C. (1993) Average Platelet Volume in Diabetes Mellitus. $Q J$ $M e, 86,739-742$.

[15] Trip, M.D., Cats, V.K., van Copelle, F.J.K., et al. (1990) Platelet Hyperactivity and Prognosis in Survivors of Myocardial Infarction. The New England Journal of Medicine, 322, 15491554. http://dx.doi.org/10.1056/NEJM199005313222201

[16] Karan, A., Guray, U., Demirkan, B., et al. (2013) Average Platelet Volume and Extent of Coronary Atherosclerosis in Patients with Stable Coronary Artery Disease. Turk Kardiyol Dem Ars, 41, 45-50. http://dx.doi.org/10.5543/tkda.2013.26235

[17] Pizzulli, L., Yong, A., Martin, J.F., et al. (1998) Changes in Platelet Size and Count in UA Compared to Stable Angina or Non Cardiac Chest Pain. European Heart Journal, 19, 80-84. http://dx.doi.org/10.1053/euhj.1997.0747

[18] Lippi, E.A., Phillips, R., Ibatson, R.M., et al. (1983) Platelet Size in Myocardial Infarction. BMJ, 287, 449-451. http://dx.doi.org/10.1136/bmj.287.6390.449 
Submit or recommend next manuscript to SCIRP and we will provide best service for you:

Accepting pre-submission inquiries through Email, Facebook, LinkedIn, Twitter, etc. A wide selection of journals (inclusive of 9 subjects, more than 200 journals)

Providing 24-hour high-quality service

User-friendly online submission system

Fair and swift peer-review system

Efficient typesetting and proofreading procedure

Display of the result of downloads and visits, as well as the number of cited articles

Maximum dissemination of your research work

Submit your manuscript at: http://papersubmission.scirp.org/

Or contact health@scirp.org 